$\Rightarrow$ SYMBIOSIS

\section{A partnership cast in iron}

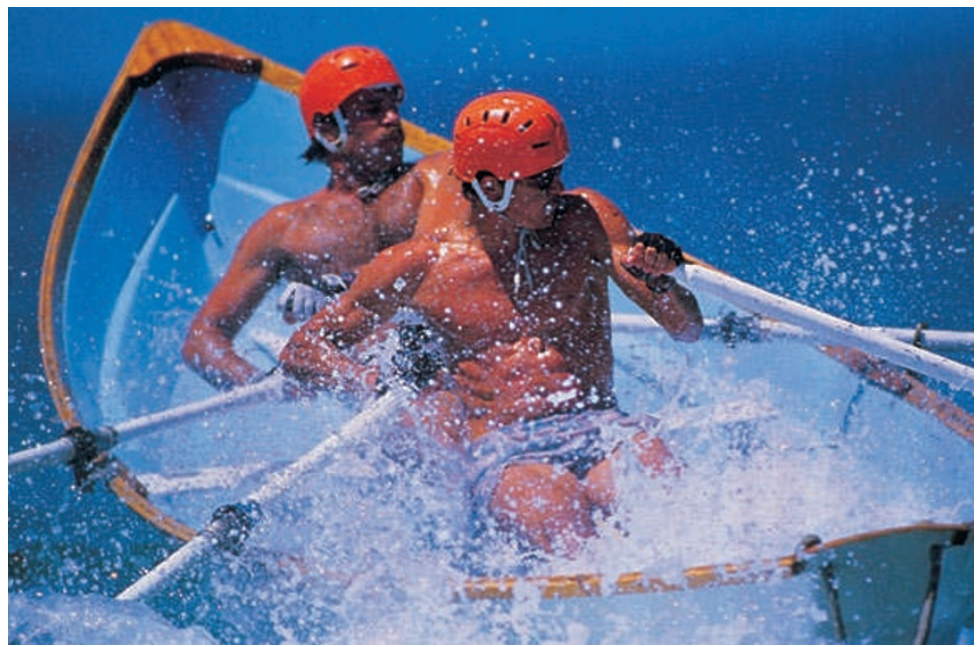

Many species of bloom-forming algae in the phytoplankton form beneficial or obligate associations with marine bacteria. Such partnerships are thought to enable the sharing of metabolites and nutrients, although a detailed understanding of most of these interactions is lacking. The acquisition of iron may be a key element that drives such associations, as iron is essential for photosynthesis and respiration but is present at extremely

a mutualistic

partnership

based on the exchange of iron and carbon has evolved between Marinobacter spp. and marine algae. low concentrations in sea water, owing to its poor solubility. Marine bacteria have evolved to produce siderophores, which are small organic molecules that bind tightly to iron and, in doing so, increase its solubility and improve its bioavailability. By contrast, phytoplankton are not known to produce siderophores or indeed to take up bacterial iron-siderophore complexes directly.
While investigating the marine bacteria that are associated with dinoflagellate and coccolithophore phytoplankton, Amin et al. observed that members of the genus Marinobacter were present in over $80 \%$ of cultures. Interestingly, the only siderophore that the authors were able to detect in the mutualistic Marinobacter spp. was vibrioferrin (VF), a carboxylate class of siderophore that was originally isolated from Vibrio parahaemolyticus. Unlike most other siderophores that are produced by free-living marine bacteria, VF-Fe III complexes undergo extremely facile photochemical oxidation, which leads to the breakdown of VF and is coupled with the reduction of iron from $\mathrm{Fe}$ III to Fe II. The Fe II then dissociates from the photolysed VF and can be rapidly reoxidized to form an inorganic ferric hydrolysis species (termed Fe III'), which can be assimilated by algae.

The authors confirmed that the VF-Fe III complexes of Marinobacter spp. were sensitive to light, with photolysis rates that were 10-20-fold higher than those for other siderophores. Using radiolabelled VF- ${ }^{55} \mathrm{Fe}$ III complexes, they found that, following photolysis of VF-Fe III in attenuated sunlight, assimilation of iron by the phytoplankton-associated Marinobacter spp. increased by $70 \%$ and, importantly, this also led to a 20 -fold increase in iron assimilation in the dinoflagellate Scrippsiella trochoidea. Although such an arrangement might be expected to be disadvantageous to the Marinobacter spp., Amin et al. observed that the growth of Marinobacter spp. in binary culture with $S$. trochoidea was dependent on algal growth - no growth was observed for bacteria incubated in algal culture media as a monoculture - suggesting that growth of Marinobacter spp. depends on organic matter that is produced by phytoplankton.

These data suggest that a mutualistic partnership based on the exchange of iron and carbon has evolved between Marinobacter spp. and marine algae. It will be interesting to see whether similar mechanisms are used to support other symbiotic relationships in environments where iron is a scarce and valuable commodity.

Andrew Jermy

ORIGINAL RESEARCH PAPER Amin, S. A. et al. Photolysis of iron-siderophore chelates promotes bacterial-algal mutualism. Proc. Natl Acad. Sci USA 23 Sep 2009 (doi:10.1073/pnas.0905512106) 